ESAIM: PROCEEDINGS AND SURVEYS, September 2014, Vol. 45, p. 447-455

J.-S. Dhersin, Editor

\title{
THE EVOLUTION OF THE LOCAL INDUCTION APPROXIMATION FOR A REGULAR POLYGON*
}

\author{
FRANCISCO DE LA HOZ ${ }^{1}$ AND LUIS VEGA ${ }^{2}$
}

\begin{abstract}
In this paper, we consider the so-called local induction approximation (LIA):

$$
\mathbf{X}_{t}=\mathbf{X}_{s} \wedge \mathbf{X}_{s s}
$$

where $\wedge$ is the usual cross product, and $s$ denotes the arc-length parametrization. We study its evolution, taking planar regular polygons of $M$ sides as initial data. Assuming uniqueness and bearing in mind the invariances and symmetries of the problem, we are able to fully characterize, by algebraic means, $\mathbf{X}(s, t)$ and its derivative, the tangent vector $\mathbf{T}(s, t)$, at times $t$ which are rational multiples of $2 \pi / M^{2}$. We show that the values at those instants are intimately related to the generalized quadratic Gauß sums.
\end{abstract}

Résumé. Dans cette courte note, nous considérons l'approximation d'induction locale (communément appelée LIA, par ses initiales en anglais):

$$
\mathbf{X}_{t}=\mathbf{X}_{s} \wedge \mathbf{X}_{s s}
$$

où $\wedge$ symbolise le produit croisé habituel et $s$ est le paramétrage par longueur d'arc. Nous étudions son évolution, en considerant des polygones plans réguliers à $M$ côtés comme données initiales. En supposant l'unicité et en prenant en compte les invariances et les symétries du problème, nous sommes capables de caractériser complètement, par des techniques algébriques, $\mathbf{X}(s, t)$ et sa dérivée, le vecteur tangent $\mathbf{T}(s, t)$, à des instants $t$ qui sont des multiples rationnels de $2 \pi / M^{2}$. Nous montrons que les valeurs à ces instants sont intimement liées aux sommes quadratiques généralisées de Gauß.

\section{INTRODUCTION}

We consider the initial-value problem for the binormal flow

$$
\mathbf{X}_{t}=\kappa \mathbf{b}
$$

\footnotetext{
* This work was supported by MEC (Spain), with the project MTM2011-24054, and by the Basque Government, with the project IT641-13.

${ }^{1}$ Department of Applied Mathematics, Statistics and Operations Research, Faculty of Science and Technology, University of the Basque Country UPV/EHU, Barrio Sarriena S/N, 48940 Leioa, Spain. e-mail: francisco.delahoz@ehu.es

2 Department of Mathematics, Faculty of Science and Technology, University of the Basque Country UPV/EHU, Barrio Sarriena S/N, 48940 Leioa, Spain. BCAM - Basque Center for Applied Mathematics, Alameda de Mazarredo 14, 48009 Bilbao, Spain. e-mail: luis.vega@ehu.es; lvega@bcamath.org

(C) EDP Sciences, SMAI 2014
} 
where $\kappa$ is the curvature and $\mathbf{b}$ the binormal component of the Frenet-Serret formulae:

$$
\left(\begin{array}{l}
\mathbf{T} \\
\mathbf{n} \\
\mathbf{b}
\end{array}\right)_{s}=\left(\begin{array}{ccc}
0 & \kappa & 0 \\
-\kappa & 0 & \tau \\
0 & -\tau & 0
\end{array}\right) \cdot\left(\begin{array}{l}
\mathbf{T} \\
\mathbf{n} \\
\mathbf{b}
\end{array}\right)
$$

observe that the torsion $\tau$ does not appear explicitly in (1). This geometric flow can be expressed as

$$
\mathbf{X}_{t}=\mathbf{X}_{s} \wedge \mathbf{X}_{s s}
$$

where $\wedge$ is the usual cross-product, $t$ is the time, and $s$ is the arc-length parameter. It appeared for the first time in 1906 [11], and was rederived in 1965 [1] as an approximation of the dynamics of a vortex filament under the Euler equations. It is also known as the local induction approximation (LIA), or the Vortex Filament Equation (VFE). Since the tangent vector $\mathbf{T}=\mathbf{X}_{s}$ remains with constant length, we can assume that $\mathbf{T} \in \mathbb{S}^{2} \forall t$, where $\mathbb{S}^{2}$ is the unit sphere in $\mathbb{R}^{3}$. Differentiating (3), we get the so-called Schrödinger map equation on the sphere:

$$
\mathbf{T}_{t}=\mathbf{T} \wedge \mathbf{T}_{s s}
$$

Even if the solutions of (3)-(4) for an initial datum with a corner are well understood, both from a theoretical $[2-5,9]$ and from a numerical point of view $[6,7]$; nothing had been done for more general initial data with several corners. However, in a recently submitted paper [8], we have considered for the first time the evolution of (3)-(4), taking a planar regular polygon of $M$ sides as the initial datum.

In this paper, we briefly announce some important results of [8]. Its structure is as follows. In Section 2 , we apply a modified version of the Hasimoto transformation [10], in order to relate (3)-(4) and the nonlinear Schrödinger (NLS) equation. In Section 3, assuming uniqueness and relying on the fact that the NLS equation is Galilean-invariant, we are able to explain, by using generalized quadratic Gauß sums, the structure of $\mathbf{X}(s, t)$ and $\mathbf{T}(s, t)$ at times $t$ that are rational multiples of $2 \pi / M^{2}$. In Section 4 , we actually recover $\mathbf{X}(s, t)$ and $\mathbf{T}(s, t)$ at those times. In Section 5, we simulate numerically (3)-(4), comparing the numerical results with those obtained algebraically in Section 4. Finally, in Section 6, we draw the main conclusions, pointing out some most recent research on the subject.

\section{The Hasimoto transformation}

A central point of this paper is the natural connection between (3)-(4) and the NLS equation. Indeed, applying the Hasimoto transformation [10]:

$$
\psi(s, t)=\kappa(s, t) \exp \left(i \int_{0}^{s} \tau\left(s^{\prime}, t\right) d s^{\prime}\right)
$$

$\psi$ satisfies

$$
\psi_{t}=i \psi_{s s}+i\left(\frac{1}{2}\left(|\psi|^{2}+A(t)\right)\right) \psi
$$

where $A(t)$ is a certain real constant that depends on time. Nevertheless, for our purposes, it is not convenient to work with the torsion $\tau$. Instead, we consider another version of the Frenet-Serret trihedron:

$$
\left(\begin{array}{c}
\mathbf{T} \\
\mathbf{e}_{1} \\
\mathbf{e}_{2}
\end{array}\right)_{s}=\left(\begin{array}{ccc}
0 & \alpha & \beta \\
-\alpha & 0 & 0 \\
-\beta & 0 & 0
\end{array}\right) \cdot\left(\begin{array}{c}
\mathbf{T} \\
\mathbf{e}_{1} \\
\mathbf{e}_{2}
\end{array}\right),
$$

for some vectors $\mathbf{e}_{1}$ and $\mathbf{e}_{2}$ that form an orthonormal base with $\mathbf{T}$. In this case, the Hasimoto transformation takes the form

$$
\psi \equiv \alpha+i \beta
$$


Bearing in mind (7), it is not too complicate to show [8] that this new $\psi$ also satisfies (6).

A very important property of the NLS equation is the fact that it is invariant by the Galilean transformations: if $\psi$ is a solution of (6), so is

$$
\tilde{\psi}_{k}(s, t) \equiv e^{i k s-i k^{2} t} \psi(s-2 k t, t), \quad \forall k, t \in \mathbb{R} .
$$

\section{A solution of $\mathbf{X}_{t}=\mathbf{X}_{s} \wedge \mathbf{X}_{s s}$ FOr a Regular polygon}

An $M$-sided regular polygon $\mathbf{X}(s, 0)$ can be regarded as a planar curve whose curvature is a sum of Dirac deltas:

$$
\kappa(s)=\frac{2 \pi}{M} \sum_{k=-\infty}^{\infty} \delta\left(s-\frac{2 \pi k}{M}\right) .
$$

Since $\mathbf{X}(s, 0)$ is planar, its torsion is zero; hence, we have $\psi(s, 0) \equiv \kappa(s)$, i.e.,

$$
\psi(s, 0)=\frac{2 \pi}{M} \sum_{k=-\infty}^{\infty} \delta\left(s-\frac{2 \pi k}{M}\right)
$$

$\psi(s, 0)$ satisfies $\psi(s, 0)=\tilde{\psi}_{M k}(s, 0) \equiv e^{i M k s} \psi(s, 0), \forall k \in \mathbb{Z}$. Hence, bearing in mind the Galilean transformations (9) and assuming uniqueness,

$$
\psi(s, t)=\tilde{\psi}_{M k}(s, t) \equiv e^{i M k s-i(M k)^{2} t} \psi(s-2 M k t, t), \quad \forall k \in \mathbb{Z}, \forall t \in \mathbb{R} .
$$

In general, the assumption of uniqueness plays an essential role along this paper. Indeed, both $\psi(s, t)$ and $\tilde{\psi}_{M k}(s, t)$ satisfy formally (6). Therefore, since both solutions of $(6), \psi(s, t)$ and $\tilde{\psi}_{M k}(s, t)$, have the same initial data at $t=0,(12)$ holds only if uniqueness is guaranteed. This also implies that the $j$-th Fourier coefficients of $\psi(s, t)$ and $\tilde{\psi}_{M k}(s, t)$ are identical:

$$
\begin{aligned}
\hat{\psi}(j, t) & =\frac{M}{2 \pi} \int_{0}^{2 \pi / M} e^{-i M j s} \psi(s, t) d s \\
& =\frac{M}{2 \pi} \int_{0}^{2 \pi / M} e^{-i M j s} \tilde{\psi}_{M k}(s, t) d s \\
& =\frac{M}{2 \pi} \int_{0}^{2 \pi / M} e^{-i M j s}\left[e^{i M k s-i(M k)^{2} t} \psi(s-2 M k t, t)\right] d s \\
& =\frac{M e^{-i(M k)^{2} t-i M(j-k)(2 M k t)}}{2 \pi} \int_{0}^{2 \pi / M} e^{-i M(j-k) s} \psi(s, t) d s \\
& =e^{-i(M k)^{2} t-i M(j-k)(2 M k t)} \hat{\psi}(j-k, t) .
\end{aligned}
$$

This identity holds for all $j$ and $k$. In particular, evaluating both sides at $j=k$ :

$$
\hat{\psi}(k, t)=e^{-i(M k)^{2} t} \hat{\psi}(0, t)
$$

so $\psi$ can be expressed as

$$
\psi(s, t)=\hat{\psi}(0, t) \sum_{k=-\infty}^{\infty} e^{-i(M k)^{2} t+i M k s},
$$

where $\hat{\psi}(0, t)$ is a constant that depends on time and that has to be chosen in such a way that the corresponding $\mathbf{X}$ and $\mathbf{T}$ are periodic of period $2 \pi$ for a fixed $t$. For instance, when $t=0$, we have trivially $\hat{\psi}(0,0)=1$. 
Moreover, combining (11) and (15), we get the following well-known identity:

$$
\sum_{k=-\infty}^{\infty} e^{i(M k) s} \equiv \frac{2 \pi}{M} \sum_{k=-\infty}^{\infty} \delta\left(s-\frac{2 \pi k}{M}\right) .
$$

Let us evaluate (15) at $t=t_{p q} \equiv\left(2 \pi / M^{2}\right)(p / q)$, where $p \in \mathbb{Z}, q \in \mathbb{N}$, and we can suppose without loss of generality that $\operatorname{gcd}(p, q)=1$ :

$$
\begin{aligned}
\psi\left(s, t_{p q}\right) & =\hat{\psi}\left(0, t_{p q}\right) \sum_{k=-\infty}^{\infty} e^{-i(M k)^{2} 2 \pi p /\left(M^{2} q\right)+i M k s} \\
& =\hat{\psi}\left(0, t_{p q}\right) \sum_{k=-\infty}^{\infty} e^{-2 \pi i(p / q) k^{2}+i M k s} \\
& =\hat{\psi}\left(0, t_{p q}\right) \sum_{l=0}^{q-1} \sum_{k=-\infty}^{\infty} e^{-2 \pi i(p / q)(q k+l)^{2}+i M(q k+l) s} \\
& =\hat{\psi}\left(0, t_{p q}\right) \sum_{l=0}^{q-1} e^{-2 \pi i(p / q) l^{2}+i M l s} \sum_{k=-\infty}^{\infty} e^{i M q k s} .
\end{aligned}
$$

Using the identity (16),

$$
\begin{aligned}
\psi\left(s, t_{p q}\right) & =\frac{2 \pi}{M q} \hat{\psi}\left(0, t_{p q}\right) \sum_{l=0}^{q-1} e^{-2 \pi i(p / q) l^{2}+i M l s} \sum_{k=-\infty}^{\infty} \delta\left(s-\frac{2 \pi k}{M q}\right) \\
& =\frac{2 \pi}{M q} \hat{\psi}\left(0, t_{p q}\right) \sum_{l=0}^{q-1} \sum_{k=-\infty}^{\infty} e^{-2 \pi i(p / q) l^{2}+i M l(2 \pi k / M q)} \delta\left(s-\frac{2 \pi k}{M q}\right) \\
& =\frac{2 \pi}{M q} \hat{\psi}\left(0, t_{p q}\right) \sum_{k=-\infty}^{\infty} \sum_{l=0}^{q-1} e^{-2 \pi i(p / q) l^{2}+2 \pi i(k / q) l} \delta\left(s-\frac{2 \pi k}{M q}\right) \\
& =\frac{2 \pi}{M q} \hat{\psi}\left(0, t_{p q}\right) \sum_{k=-\infty}^{\infty} \sum_{m=0}^{q-1} \sum_{l=0}^{q-1} e^{-2 \pi i(p / q) l^{2}+2 \pi i(m / q) l} \delta\left(s-\frac{2 \pi k}{M}-\frac{2 \pi m}{M q}\right) \\
& =\frac{2 \pi}{M q} \hat{\psi}\left(0, t_{p q}\right) \sum_{k=-\infty}^{\infty} \sum_{m=0}^{q-1} G(-p, m, q) \delta\left(s-\frac{2 \pi k}{M}-\frac{2 \pi m}{M q}\right)
\end{aligned}
$$

where

$$
G(a, b, c)=\sum_{l=0}^{c-1} e^{2 \pi i\left(a l^{2}+b l\right) / c}, \quad a, b \in \mathbb{Z}, c \in \mathbb{Z}-\{0\},
$$

denotes a generalized quadratic Gauß sum. For our purposes, an important property of these sums is that

$$
|G(-p, m, q)|= \begin{cases}\sqrt{q}, & \text { if } q \text { is odd, } \\ \sqrt{2 q}, & \text { if } q \text { is even and } q / 2 \equiv m \bmod 2 \\ 0, & \text { if } q \text { is even and } q / 2 \not \equiv m \bmod 2\end{cases}
$$


Therefore, $\psi(s, t)$ has evolved from $M$ Dirac deltas in $[0,2 \pi)$ at $t=0$, to $M q$ deltas in $[0,2 \pi)$ at $t_{p q}$, for $q$ odd; and $M q / 2$ deltas in $[0,2 \pi)$ at $t_{p q}$, for $q$ even. Furthermore, from (20),

$$
G(-p, m, q)=\left\{\begin{array}{lll}
\sqrt{q} e^{i \theta_{m}}, & \text { if } q \text { is odd } \\
\sqrt{2 q} e^{i \theta_{m}}, & \text { if } q \text { is even and } q / 2 \equiv m \quad \bmod 2 \\
0, & \text { if } q \text { is even and } q / 2 \not \equiv m \quad \bmod 2
\end{array}\right.
$$

for a certain angle $\theta_{m}$ that depends on $m$ (and, of course, on $p$ and $q$, too). Introducing (21) into (18), and restricting ourselves to $k=0$, i.e., $\left[0, \frac{2 \pi}{M}\right)$, we conclude that

$$
\psi\left(s, t_{p q}\right)= \begin{cases}\frac{2 \pi}{M \sqrt{q}} \hat{\psi}\left(0, t_{p q}\right) \sum_{m=0}^{q-1} e^{i \theta_{m}} \delta\left(s-\frac{2 \pi m}{M q}\right), & \text { if } q \text { odd }, \\ \frac{2 \pi}{M \sqrt{\frac{q}{2}}} \hat{\psi}\left(0, t_{p q}\right) \sum_{\substack{q / 2-1 \\ m=0}}^{i \theta_{2 m+1} \delta\left(s-\frac{4 \pi m+2 \pi}{M q}\right),} & \text { if } q / 2 \text { odd } \\ \frac{2 \pi}{M \sqrt{\frac{q}{2}}} \hat{\psi}\left(0, t_{p q}\right) \sum_{m=0}^{q / 2-1} e^{i \theta_{2 m}} \delta\left(s-\frac{4 \pi m}{M q}\right), & \text { if } q / 2 \text { even. }\end{cases}
$$

The coefficients multiplying the Dirac deltas are in general not real, except for $t=0$ and $t_{1,2}=\pi / M^{2}$. Therefore, $\psi\left(s, t_{p q}\right)$ does not correspond to a planar polygon, but to a skew polygon with $M q$ sides, for $q$ odd; and to a skew polygon with $M q / 2$ sides, for $q$ even. Since the Dirac deltas are equally spaced at a time $t=t_{p q}$, the length of the sides is the same. Finally, $\hat{\psi}\left(0, t_{p q}\right)$ has to be determined in such a way that the polygon is closed.

\section{Recovering $\mathbf{X}$ and $\mathbf{T}$ from $\psi$ at $t=t_{p q}$}

Given $\psi(s, t)=\alpha(s, t)+i \beta(s, t)$, recovering $\mathbf{T}, \mathbf{e}_{1}$ and $\mathbf{e}_{2}$ from $\psi$ implies integrating

$$
\left(\begin{array}{c}
\mathbf{T} \\
\mathbf{e}_{1} \\
\mathbf{e}_{2}
\end{array}\right)_{s}=\left(\begin{array}{ccc}
0 & \alpha & \beta \\
-\alpha & 0 & 0 \\
-\beta & 0 & 0
\end{array}\right) \cdot\left(\begin{array}{c}
\mathbf{T} \\
\mathbf{e}_{1} \\
\mathbf{e}_{2}
\end{array}\right)
$$

As we have seen in the previous section, at a time $t_{p q}=\left(2 \pi / M^{2}\right)(p / q), \psi\left(s, t_{p q}\right)$ is a sum of $M q$ (if $q$ odd) or $M q / 2$ (if $q$ even) equally spaced Dirac deltas, that corresponds to a skew polygon $\mathbf{X}\left(s, t_{p q}\right)$ of $M q$ or $M q / 2$ sides. To integrate (23), we have to understand the transition from one side of the polygon to the next one. In order to do that, we reduce ourselves, without loss of generality, to a certain $\psi$ formed by a single Dirac delta located at $s=0$, i.e, $\psi(s)=(a+i b) \delta(s)$, so we have to integrate

$$
\left(\begin{array}{c}
\mathbf{T} \\
\mathbf{e}_{1} \\
\mathbf{e}_{2}
\end{array}\right)_{s}=\left(\begin{array}{ccc}
0 & a \delta(s) & b \delta(s) \\
-a \delta(s) & 0 & 0 \\
-b \delta(s) & 0 & 0
\end{array}\right) \cdot\left(\begin{array}{c}
\mathbf{T} \\
\mathbf{e}_{1} \\
\mathbf{e}_{2}
\end{array}\right)=\delta(s) \mathbf{A} \cdot\left(\begin{array}{c}
\mathbf{T} \\
\mathbf{e}_{1} \\
\mathbf{e}_{2}
\end{array}\right)
$$

The solution of this equation is

$$
\left(\frac{\mathbf{T}\left(0^{+}\right)^{T}}{\mathbf{e}_{1}\left(0^{+}\right)^{T}}\right)=\exp (\mathbf{A}) \cdot\left(\frac{\mathbf{T}\left(0^{-}\right)^{T}}{\mathbf{e}_{2}\left(0^{+}\right)^{T}}\right)
$$


where the rotation matrix $\exp (\mathbf{A})$ can be computed explicitly. Expressing $a+i b$ in polar form, $a+i b \equiv \rho e^{i \theta}$, we get

$$
\exp (\mathbf{A})=\left(\begin{array}{ccc}
\cos (\rho) & \sin (\rho) \cos (\theta) & \sin (\rho) \sin (\theta) \\
-\sin (\rho) \cos (\theta) & \cos (\rho) \cos ^{2}(\theta)+\sin ^{2}(\theta) & {[\cos (\rho)-1] \cos (\theta) \sin (\theta)} \\
-\sin (\rho) \sin (\theta) & {[\cos (\rho)-1] \cos (\theta) \sin (\theta)} & \cos (\rho) \sin ^{2}(\theta)+\cos ^{2}(\theta)
\end{array}\right)
$$

Since the scalar product between two vectors is rotation-invariant, choosing the orthonormal base $\left\{\mathbf{T}\left(0^{-}\right)\right.$, $\left.\mathbf{e}_{1}\left(0^{-}\right), \mathbf{e}_{2}\left(0^{-}\right)\right\}$to form the identity matrix $\mathbf{I}$, we conclude from $(26)$ that $\mathbf{T}\left(0^{-}\right) \cdot \mathbf{T}\left(0^{+}\right)=\cos (\rho)$, i.e., $\rho$ is precisely the angle formed by $\mathbf{T}\left(0^{-}\right)$and $\mathbf{T}\left(0^{+}\right)$.

Coming back to the general form of $\psi$, we have to integrate (24) $M q$ or $M q / 2$ times to obtain a closed skew, i.e., non-planar polygon with $M q$ or $M q / 2$ sides. But, according to (22), in $\left[0, \frac{2 \pi}{M}\right)$,

$$
\psi\left(s, t_{p q}\right)= \begin{cases}\sum_{m=0}^{q-1}\left(\alpha_{m}+i \beta_{m}\right) \delta\left(s-\frac{2 \pi m}{M q}\right), & \text { if } q \text { odd } \\ \sum_{\substack{m=0 \\ q / 2-1}}\left(\alpha_{2 m+1}+i \beta_{2 m+1}\right) \delta\left(s-\frac{4 \pi m+2 \pi}{M q}\right), & \text { if } q / 2 \text { odd } \\ \sum_{m=0}^{q / 2-1}\left(\alpha_{2 m}+i \beta_{2 m}\right) \delta\left(s-\frac{4 \pi m}{M q}\right), & \text { if } q / 2 \text { even }\end{cases}
$$

where

$$
\left|\alpha_{m}+i \beta_{m}\right|=\rho= \begin{cases}\frac{2 \pi}{M \sqrt{q}} \hat{\psi}\left(0, t_{p q}\right), & \text { if } q \text { is odd, } \\ \frac{2 \pi}{M \sqrt{\frac{q}{2}}} \hat{\psi}\left(0, t_{p q}\right), & \text { if } q \text { is even and } q / 2 \equiv m \bmod 2, \\ 0, & \text { if } q \text { is even and } q / 2 \not \equiv m \bmod 2,\end{cases}
$$

so we conclude that, at any time $t_{p q}$, the angle $\rho$ between two adjacent sides is constant. Furthermore, the structure of the polygon is completely determined by the angles $\theta_{m}$ appearing in the generalized quadratic Gaussian sum, where $\alpha_{m}+i \beta_{m}=\rho e^{i \theta_{m}}$.

Let $\mathbf{M}_{m}$ be the rotation matrix corresponding to each $\left(\alpha_{m}+i \beta_{m}\right) \delta(s)=e^{i \theta_{m}} \delta(s)$ in (22). If $\alpha_{m}+i \beta_{m} \equiv 0$, $\mathbf{M}_{m}$ is simply the identity matrix $\mathbf{I}$ and can be ignored. Otherwise, from (26),

$$
\mathbf{M}_{m}=\left(\begin{array}{ccc}
\cos (\rho) & \sin (\rho) \cos \left(\theta_{m}\right) & \sin (\rho) \sin \left(\theta_{m}\right) \\
-\sin (\rho) \cos \left(\theta_{m}\right) & \cos (\rho) \cos ^{2}\left(\theta_{m}\right)+\sin ^{2}\left(\theta_{m}\right) & {[\cos (\rho)-1] \cos \left(\theta_{m}\right) \sin \left(\theta_{m}\right)} \\
-\sin (\rho) \sin \left(\theta_{m}\right) & {[\cos (\rho)-1] \cos \left(\theta_{m}\right) \sin \left(\theta_{m}\right)} & \cos (\rho) \sin ^{2}\left(\theta_{m}\right)+\cos ^{2}\left(\theta_{m}\right)
\end{array}\right)
$$

Therefore,

$$
\left(\frac{\mathbf{T}\left(\frac{2 \pi k}{M q}^{+}\right)^{T}}{\mathbf{e}_{1}\left(\frac{2 \pi k}{M q}\right)^{T}}\right)=\mathbf{M}_{k} \cdot \mathbf{M}_{k-1} \cdot \ldots \mathbf{M}_{1} \cdot \mathbf{M}_{0} \cdot\left(\frac{\mathbf{T}\left(0^{-}\right)^{T}}{\mathbf{e}_{2}\left(\frac{2 \pi k}{M q}\right)^{+}}\right), \quad \forall k \in \mathbb{N} .
$$

In order that the polygon is closed, we have to choose $\rho$ in (28) in such a way that $\mathbf{T}, \mathbf{e}_{1}$ and $\mathbf{e}_{2}$ are periodic, i.e.,

$$
\left(\frac{\mathbf{T}\left(2 \pi^{-}\right)^{T}}{\mathbf{e}_{1}\left(2 \pi^{-}\right)^{T}}\right)=\left(\frac{\mathbf{T}\left(0^{-}\right)^{T}}{\mathbf{e}_{2}\left(2 \pi^{-}\right)^{T}}\right)=\left(\frac{\mathbf{e}_{1}\left(0^{-}\right)^{T}}{\mathbf{e}_{2}\left(0^{-}\right)^{T}}\right)
$$

Evaluating (30) at $k=M q-1,(31)$ is equivalent to imposing that

$$
\mathbf{M}_{M q-1} \cdot \mathbf{M}_{M q-2} \cdot \ldots \cdot \mathbf{M}_{1} \cdot \mathbf{M}_{0} \equiv \mathbf{I} .
$$


Let us define

$$
\mathbf{M}=\mathbf{M}_{q-1} \cdot \mathbf{M}_{q-2} \cdot \ldots \cdot \mathbf{M}_{1} \cdot \mathbf{M}_{0} .
$$

From (32), since $\mathbf{M}_{k+q} \equiv \mathbf{M}_{k} \forall k, \mathbf{M}$ is an $M$-th root of the identity matrix $\mathbf{I}$. Moreover, it is also a rotation matrix that induces a rotation of $2 \pi / M$ degrees around a certain rotation axis. Therefore, we have to choose $\rho$ in order that any of the following equivalent properties is satisfied:

$$
\begin{aligned}
\operatorname{Tr}(\mathbf{M}) & =1+2 \cos \left(\frac{2 \pi}{M}\right), \\
\lambda(\mathbf{M}) & =\left\{1, e^{2 \pi i / M}, e^{-2 \pi i / M}\right\},
\end{aligned}
$$

were $\operatorname{Tr}(\mathbf{M})$ and $\lambda(\mathbf{M})$ denote the trace and the spectrum of $\mathbf{M}$, respectively. We have worked with the trace, because it is algebraically easier. By means of a symbolic manipulator, it is not difficult to calculate $\rho$, or more precisely $\cos (\rho)$, for some small values of $q$ (v.g. $q=3,4,5,6,8)$, in order that (34) holds. The expressions obtained algebraically for $\cos (\rho)$ for those values of $q$ strongly suggest that, for any $q$ and for any $p$ coprime with it, the only possible real value for $\cos (\rho)$ is

$$
\cos (\rho)= \begin{cases}2 \cos ^{2 / q}\left(\frac{\pi}{M}\right)-1, & \text { if } q \text { is odd } \\ 2 \cos ^{4 / q}\left(\frac{\pi}{M}\right)-1, & \text { if } q \text { is even }\end{cases}
$$

Although giving a universal proof that this formula holds for any $q$ seems complicate, we have checked it for a few more $q$. Moreover, it is absolutely in agreement with our numerical simulations (see [8] for more details). Therefore, we think that there is concluding evidence that this formula is valid for any $q$. Hence, we are able to determine the value of $\hat{\psi}\left(0, t_{p q}\right)$ in $(15)$ :

$$
\hat{\psi}\left(0, t_{p q}\right)= \begin{cases}\frac{M \sqrt{q}}{2 \pi} \arccos \left(2 \cos ^{2 / q}\left(\frac{\pi}{M}\right)-1\right), & \text { if } q \text { is odd } \\ \frac{M \sqrt{\frac{q}{2}}}{2 \pi} \arccos \left(2 \cos ^{4 / q}\left(\frac{\pi}{M}\right)-1\right), & \text { if } q \text { is even }\end{cases}
$$

After obtaining completely $\psi\left(s, t_{p q}\right)$, we have integrated (23) up to a rigid moment, but the symmetries of (3)-(4) allow to compute the correct rotation of $\mathbf{X}$ and $\mathbf{T}$ at $t=t_{p q}$. Thus, $\mathbf{T}$ may be fully determined by algebraic means, while we have been able to determine $\mathbf{X}$ up to a vertical movement.

\section{NumERICAL EXPERIMENTS}

We have simulated numerically a combination of (3) and (4),

$$
\left\{\begin{array}{l}
\mathbf{X}_{t}=\mathbf{T} \wedge \mathbf{T}_{s} \\
\mathbf{T}_{t}=\mathbf{T} \wedge \mathbf{T}_{s s}
\end{array}\right.
$$

using a fourth-order Runge-Kutta scheme in time, together with a pseudo-spectral discretization directly in space. More precisely, since we deal with periodic solutions in $s \in[0,2 \pi)$, we have simulated the evolution of $\mathbf{X}=\left(X_{1}, X_{2}, X_{3}\right)$ and $\mathbf{T}=\left(T_{1}, T_{2}, T_{3}\right)$ at $N$ equally spaced nodes $s_{j}=2 \pi j / N, j=0, \ldots, N-1$.

In Figure 1, we show the numerically obtained $\mathbf{T}_{\text {num }}$ versus the algebraically obtained $\mathbf{T}_{\text {alg }}$, conveniently rotated, for $M=3, s \in[0,2 \pi)$, at $t_{1,3}=\frac{2 \pi}{27}$. We have taken $3 \times 4096$ frequencies. $T_{1}$ appears in blue, $T_{2}$ in green, $T_{3}$ in red. In $\mathbf{T}_{n u m}$, the Gibbs phenomenon is clearly visible. Otherwise, the maximum discrepancy between $\mathbf{T}_{\text {num }}$ and $\mathbf{T}_{\text {alg }}$ at the 27 points indicated by the black circles is $6.3869 \cdot 10^{-9}$.

With respect to $\mathbf{X}$, we are able to fully determine it algebraically, except for a vertical movement. Therefore, in order to completely specify $\mathbf{X}$ at a given time, we would need to give the height of one point or, more 

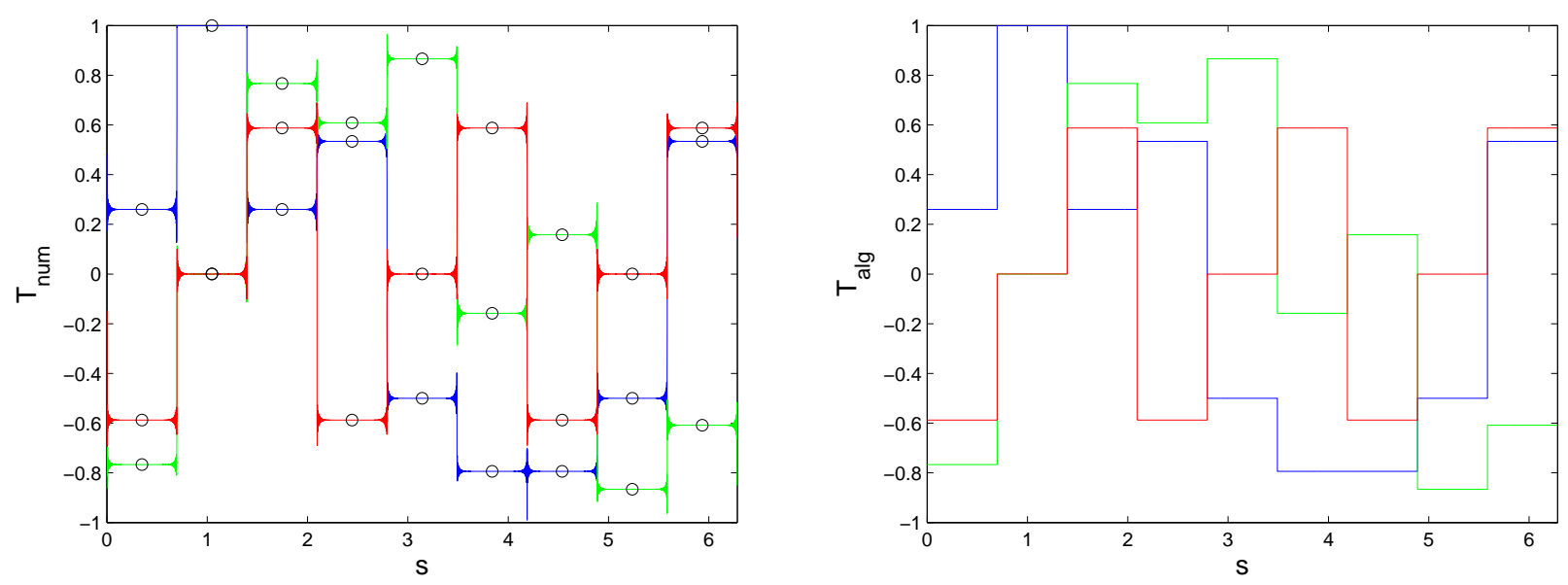

FiguRE 1. $\mathbf{T}_{\text {num }}$ versus $\mathbf{T}_{\text {alg }}$, for $M=3$, at $t_{1,3}=\frac{2 \pi}{27}$.

conveniently, the height $h(t)$ of the mass center, which is precisely the mean of all the values $X_{3}\left(s_{j}, t\right)$ :

$$
h(t)=\frac{1}{N} \sum_{j=0}^{N-1} X_{3}\left(s_{j}, t\right) ;
$$

remember that, thanks to the symmetries of the problem, the mean of both $X_{1}\left(s_{j}, t\right)$ and $X_{2}\left(s_{j}, t\right)$ is always zero. There is concluding numerical evidence that $h(t)$ grows linearly with $t$, i.e.,

$$
h(t)=\frac{h\left(2 \pi / M^{2}\right)}{2 \pi / M^{2}} t=c_{M} t
$$

where $c_{M}$ is a constant that depends on the number of initial sides $M$. Furthermore, after adjusting $c_{M}$, a very good agreement is also found between $\mathbf{X}_{n u m}$ and $\mathbf{X}_{a l g}$. For instance, we have compared the $\mathbf{X}_{a l g}$ and $\mathbf{X}_{n u m}$ corresponding to Figure (1). Taking $c_{3}=0.7645$,

$$
\left\|\mathbf{X}_{\text {alg }}+\left(0,0, c_{3} t_{1,3}\right)-\mathbf{X}_{n u m}\right\|_{\mathcal{L}^{\infty}}=3.9325 \cdot 10^{-4}
$$

Figure 2 shows $\mathbf{X}_{\text {num }}$. The nine equal-lengthed sides are clearly visible.

\section{Conclusions And most RECEnt ReSEARCH}

In this paper, we have studied the evolution of (3)-(4), for a regular planar polygonal of $M$ sides as initial datum. The algebraic calculations, backed by complete numerical simulations, suggest very strongly that $\mathbf{X}(s, t)$ is a polygon at times which are rational multiples of $2 \pi / M^{2}$, i.e., $t_{p q}=\left(2 \pi / M^{2}\right)(p / q)$, with the number of sides depending on $q$, while $\mathbf{T}(s, t)$ is piecewise constant at those times.

This paper raises multiple questions. We are currently conducting research on the subject and our priority is to give sense to our solutions from an analytical point of view. Moreover, while our $\mathbf{X}(s, t)$, obtained algebraically for rational times, can be extended by continuity to all $t \in \mathbb{R}$, it is not clear how to give sense to $\mathbf{T}(s, t)$ at irrational times. In this line, we have found recently that the evolution of the polygon from $t=0$ to $t=\varepsilon \ll 1$ can be conveniently explained by means of the one-corner model [9], i.e., we can say, loosely speaking, that the corners do not see each other at infinitesimal times. As a consequence, we have been able to obtain numerically $c_{M}$ in (40) using exclusively the results in [9]. We will explain all this in a future paper. 


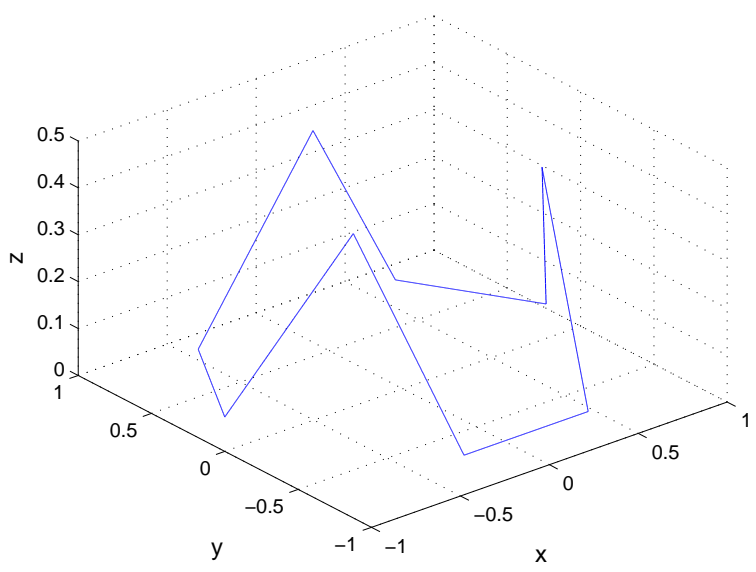

Figure 2. $\mathbf{X}_{\text {num }}$, for $M=3$, at $t_{1,3}=\frac{2 \pi}{27}$.

\section{REFERENCES}

[1] R. J. Arms and F. R. Hama. Localized-Induction Concept on a Curved Vortex and Motion of an Elliptic Vortex Ring. Phys. Fluids, 8(4):553-559, 1965.

[2] V. Banica and L. Vega. On the Stability of a Singular Vortex Dynamics. Commun. Math. Phys., 286(2):593-627, 2009.

[3] V. Banica and L. Vega. Scattering for 1D cubic NLS and singular vortex dynamics. J. Eur. Math. Soc. (JEMS), 14(1):209-253, 2012.

[4] V. Banica and L. Vega. Stability of the Self-similar Dynamics of a Vortex Filament. ARMA, 210(3):673-712, 2013.

[5] V. Banica and L. Vega. The initial value problem for the Binormal Flow with rough data. 2013. arXiv:1304.0996.

[6] T. F. Buttke. A Numerical Study of Superfluid Turbulence in the Self-Induction Approximation. J. Comput. Phys., 76(2):301326, 1998.

[7] F. de la Hoz, C. J. García-Cervera, and L. Vega. A Numerical Study of the Self-Similar Solutions of the Schrödinger Map. SIAM J. Appl. Math., 70(4):1047-1077, 2009.

[8] F. de la Hoz and L. Vega. Vortex Filament Equation for a Regular Polygon. 2013. arXiv:1304.5521.

[9] S. Gutiérrez, J. Rivas, and L. Vega. Formation of singularities and self-similar vortex motion under the localized induction approximation. Comm. PDE, 28(5-6):927-968, 2003.

[10] H. Hasimoto. A soliton on a vortex filament. J. Fluid Mech., 51(3):477-485, 1972.

[11] L. S. Da Rios. On the motion of an unbounded fluid with a vortex filament of any shape. Rend. Circ. Mat. Palermo, 22:117-135, 1906. In Italian. 